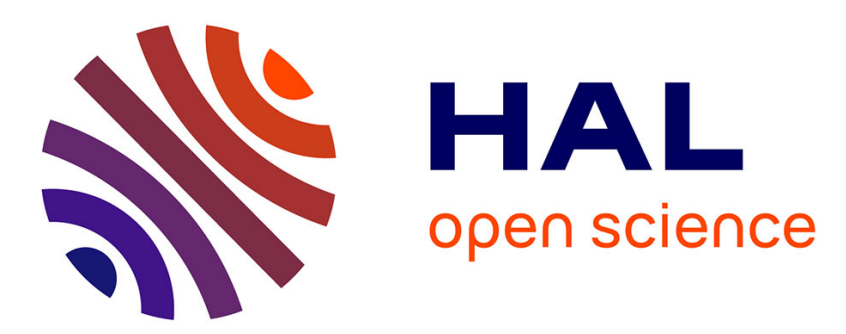

\title{
De la raison en architecture: projets et chantiers des églises Saint-Bruno de Voiron et de Grenoble au XIXe siècle
}

Cédric Avenier

\section{- To cite this version:}

Cédric Avenier. De la raison en architecture: projets et chantiers des églises Saint-Bruno de Voiron et de Grenoble au XIXe siècle. Livraisons d'Histoire de l'Architecture, 2006, 11 (1), pp.97 - 118. 10.3406/lha.2006.1036 . hal-01872647

\section{HAL Id: hal-01872647 https://hal.science/hal-01872647}

Submitted on 12 Sep 2018

HAL is a multi-disciplinary open access archive for the deposit and dissemination of scientific research documents, whether they are published or not. The documents may come from teaching and research institutions in France or abroad, or from public or private research centers.
L'archive ouverte pluridisciplinaire HAL, est destinée au dépôt et à la diffusion de documents scientifiques de niveau recherche, publiés ou non, émanant des établissements d'enseignement et de recherche français ou étrangers, des laboratoires publics ou privés. 


\section{De la raison en architecture : projets et chantiers des églises} Saint-Bruno de Voiron et de Grenoble au XIXe siècle

In: Livraisons d'histoire de l'architecture. n¹1, 1er semestre 2006. pp. 97-118.

Citer ce document / Cite this document :

Avenier Cédric. De la raison en architecture : projets et chantiers des églises Saint-Bruno de Voiron et de Grenoble au XIXe siècle. In: Livraisons d'histoire de l'architecture. $\mathrm{n}^{\circ} 11$, 1er semestre 2006. pp. 97-118.

doi : $10.3406 /$ ha.2006.1036

http://www.persee.fr/web/revues/home/prescript/article/lha_1627-4970_2006_num_11_1_1036 


\section{Abstract}

"About reason in architecture. The projects and yards of the Saint-Bruno's churches in Grenoble and Voiron in the XIXth century", by Cédric Avenier. A history of architecture based on the analysis of buildings and the study of archives sometimes enables to relativize the historical value of monuments. The Neo-Gothic Saint- Bruno's church in Voiron (1857-1871) designed by the architect Alfred Berruyer neither makes use of cast cement nor even is a perfectly representative work of the Rationalist movement. The yard was a sequence of conciliations between the building owner, the Council of the Civil Buildings and the architect. The final work reminds exactly of the features of some well-known churches erected at the same time, excluding the architectonic structure. The Neo-Romanesque SaintBruno's church in Grenoble (1869-1881) is not exclusively Alfred Berruyer's work and, is it entirely of cast cement, the lack of freestones is the only reason to be found. The winning project of the competition, Anatole de Baudot's one, was not used as a model ; the designer of the executed project, Alphonse Durand, drew his inspiration from a well-known cathedral and did not supervise the work; two rival architects, Eugène Perronnet and Alfred Berruyer, followed one another at the head of the yard and each altered the project in his own way, not to mention the liberties taken by the contractors. So the two Saint-Bruno's churches in Voiron and Grenoble turn out not to be the predicted ultimate originality. However, a reasoned interpretation of these buildings - since they are in fact representative of the XIXth century architecture - leads us to give a new definition of Rationalism, not as a scholar theory, but as the pragmatic adaptation of the production equipments to the ups and downs of the construction process.

\section{Résumé}

«De la raison en architecture. Projets et chantiers des églises Saint-Bruno de Grenoble et de Voiron au XIXe siècle », par Cédric Avenier Une histoire de l'architecture fondée sur l'analyse des édifices et l'étude des sources archivistique permet quelquefois de relativiser la valeur historique des monuments. L'église néo-gothique Saint-Bruno de Voiron (1857-1871) n'est pas en ciment moulé ni même une œuvre parfaite du courant rationaliste due à son architecte Alfred Berruyer. Le chantier fut une suite de conciliations des partis, celui du maître d'ouvrage, du conseil des bâtiments civils et de l'architecte. L'œuvre achevée renvoie traits pour traits à des églises élevées et connues à la même époque, sans en avoir la structure architectonique. L'église néo-romane Saint-Bruno de Grenoble (1869-1881) n'est pas l'œuvre du seul Alfred Berruyer et, si elle est complètement en ciment moulé, c'est à défaut de pierre de taille. Le projet vainqueur du concours, celui d'Anatole de Baudot, n'a pas servi de modèle ; l'auteur du projet réalisé, Alphonse Durand, s'est inspiré d'une cathédrale connue et n'a pas dirigé les travaux ; deux architectes rivaux, Eugène Péronnet et Alfred Berruyer, se sont succédés à la direction du chantier en apportant tour à tour des modifications ; sans compter les libertés prises par les entrepreneurs. Les deux églises Saint-Bruno de Voiron et de Grenoble ne sont pas les chefs- d'œuvre d'originalité annoncés. Cependant, une lecture raisonnée de ces chantiers, puisqu'ils sont en fait symptomatiques de l'architecture du XIXe siècle, amène à redéfinir le rationalisme non comme une théorie savante, mais comme l'adaptation pragmatique des outils de production aux aléas de la construction.

\section{Zusammenfassung}

« Über Vernunft in Architektur. Projekte und Bau der Kirchen Saint-Bruno in Grenoble und in Voiron im 19. Jahrhundert », von Cédric Avenier. Eine Geschichte der Architektur, die sich auf die Untersuchung der Gebäude und die Studie der Archivquellen stützt, wird manchmal den historischen Wert der Bauwerke relativieren müssen. Die neogotische Kirche Saint-Bruno in Voiron ist kein Gussbetonbau ; ebenso lasst sich an diesem Werk des Architekten Berruyer kein rationalistischer Zugang erkennen. Die Baugeschichte gab einfach Anlass zu etlichen Ubereinkünften zwischen den verschiedenen Parteien, dem Bauherren, dem Aufsichtsrat fur Zivilbauten (conseil des Bâtiments publics) und dem Architekten. Das fertige Bauwerk ahnelt den in der gleichen Zeit gebauten und damais bekannten Kirchen Stuck for Stück, inm fehlt nur deren architektonische Struktur. Was die neoromanische Kirche Saint-Bruno in Grenoble (1869-1881) betrifft, wo Alfred Berruyer nicht allein wirkte, verwandte man Gussbeton for die ganze Konstruktion nur aufgrund eines volligen Mangels an Quaderstein. Das Projekt von Anatole de Baudot, Gewinner des Wettbewerbs, stand dafor nicht Modell ; der Autor des durchgefohrten Projekts, 
Alphonse Durand, der sich durch eine bekannte Kathedrale inspirieren liefi, durfte das Bauwerk nicht leiten, so dass zwei konkurrierende Architekten, Eugène Peronnet und Alfred Berruyer, folgten. Beide unternahmen hintereinander neue Anderungen am Bauwerk; ganz zu schweigen von manchen Freiheiten, die sich die Bauunternehmer gestatteten. So stellen die beiden Kirchen "Saint-Bruno », in Voiron und in Grenoble, nicht die annoncierten Meisterwerke an Originalitat dar. Da diese Bauwerke sich trotzdem for die Architektur des 19. Jahrhunderts als typisch erweisen, fohrt eine vernüntige Lektüre dieser Gebàude dazu, den Rationalismus neu zu definieren, nicht als durchdachte Théorie, sondern als pragmatische Anpassung der Produktionswerkzeuge an die stàndigen Ànderungen an den Bauwerken. 
Par Cédric Avenier

\section{DE LA RAISON EN ARCHITECTURE : PROJETS ET CHANTIERS DES EGLISES SAINT-BRUNO DE VOIRON ET DE GRENOBLE AU XIX ${ }^{e}$ SIECLE}

La basilique Notre-Dame de La Salette (1852-1865, Isère) - église d'un pèlerinage déclenché dès l'apparition de la Vierge en "dame blanche " aux jeunes bergers Mélanie Calvat et Maximin Giraud l'apres-midi du 19 septembre 1846 - fut dessinée par l'architecte Alfred Berruyer (1819-1901) qui imagina, pour sa première œuvre importante, un plan basilical sans déambulatoire ni grands décors, une structure porteuse de colonnes à tambours en pierre de taille et une charpente en bois. Le chantier de cet édifice, de la taille d'une église de chef-lieu de canton, fut complexe à cause de son isolement au cour du massif montagneux de la Matheysine et de ses 1770 mètres d'altitude, et son maître d'oeuvre dut importer des matériaux et des techniques de construction à peine maîtrisés dans les grandes villes. Il fit venir la pierre de la vallée et le bois, de la forêt voisine. Une dépense de deux millions de francs s'en suivit ${ }^{1}$. La Salette est plus célèbre pour ses secrets que par ses réalités. Elle n'est pas la seule : les deux églises Saint-Bruno de Voiron et de Grenoble en Isère sont en bonne place. La première fut l'église paroissiale la plus grande et la plus chère du département de l'Isère, la seconde s'inscrit en premier sur la liste des grands concours d'architecture dans ce même département.

\section{Un contretype néo-gothique : l'église Saint-Bruno de Voiron (1857-1871)}

Charles-Auguste Questel (1807-1888), inspecteur général des Bâtiments civils, trouvait l'eglise Saint-Bruno de Voiron (ill. 1) trop grande pour cette ville et l'avait appelée dès 1861 "la grandiose église aux dispositions de cathédrale " ${ }^{2}$. La tradition veut que l'on nomme Saint-Bruno « la cathédrale de Voiron ", quand la cathédrale

1. Cédric Avenier, Ciments d'églises, semences de chretiens. Constructions religieuses et industrie cimentière en Istre, thèse de doctorat, Université de Grenoble II Pierre Mendès France, sous la direction du professeur Thierry Dufrêne, 2004, vol. I, p. 48-58, 100-102, 158, 195-196, 536, 577-580. Voir aussi de Jean-Michel Leniaud, "La basilique de La Salette : l'achat du terrain, la construction, l'érection de la chapelle en basilique mineure " dans La Salette. Apocalypse, pelerinage et littérature (1856-1996), François Angelier et Claude Langlois ed., Grenoble, éd. Jérome Million, 2000, p. 135-153 et de Philippe Dufieux, « Un architecte au service des ambitions épiscopales : Alfred Berruyer (1819-1901), diocésain de Grenoble ", dans Livraisons d'histoire de l'architecture, $n^{\circ}$ 6, 2003, p. 121-133.

2. Arch. mun. Voiron, 2 M 2, Charles Questel, Rapport sur le projet d'église a Voiron, 29 octobre 1861. 


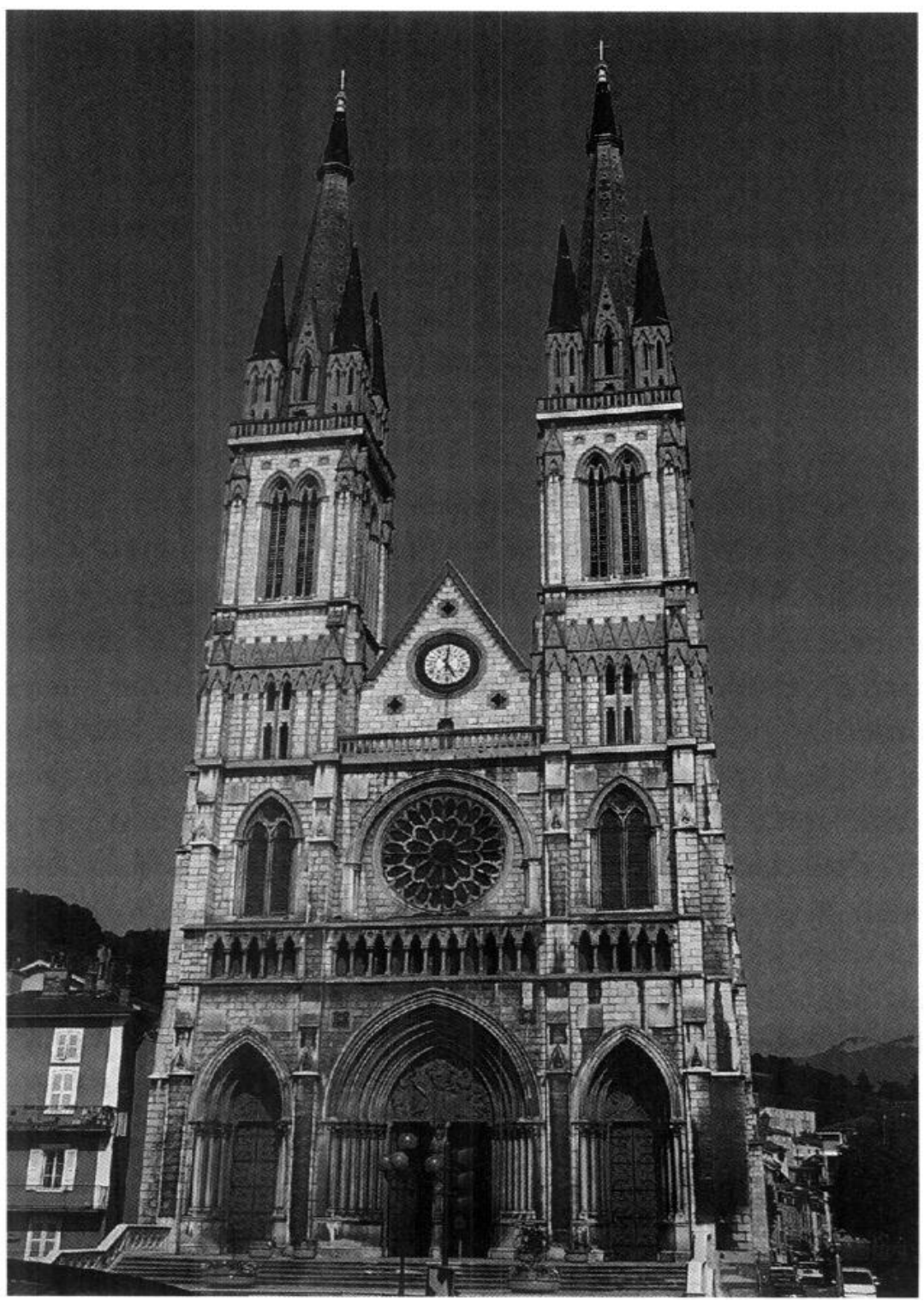

Ill. 1 : Alfred Berruyer, église Saint-Bruno de Voiron, façade principale (1857-1871). Cl. C. Avenier.

du diocèse se contente de l'appellation plus simple de " Notre-Dame de Grenoble "; elle ajoute que cette " cathédrale du XIX siècle " aurait été réalisée en collaboration avec Eugène-Emmanuel Viollet-le-Duc. On déclare enfin qu'elle serait entièrement en ciment et la première église élevée selon cette technique; avec plusieurs catégories de ciments, ocre, jaune et rose pour un bel effet polychromique. Ces attributs auraient fait d'elle une construction emblématique de la région. Son auteur, Berruyer, affirme : "Nous avons entendu des voyageurs qui en faisaient l'éloge et qui la désignait comme l'édifice moderne du Dauphiné le plus remarquable ${ }^{3}$.

3. Alfred Berruyer, “Discours de réception à l'Académie Delphinale, séance du 16 mai 1890. L'art et le style en architecture avec aperçu de l'architecture delphinale ", Bulletin de l'Académie delphinale, $1890,4^{\mathrm{c}}$ série, tome IV, 16 mai 1890 , p. 112-113. 
Bien sûr, Saint-Bruno de Voiron n'est pas une cathédrale. Les dossiers de l'administration des cultes et du diocèse de Grenoble ne renferment aucune trace de projet du genre; et Viollet-le-Duc ne s'y est pas intéressé. Elle n'est pas entièrement en ciment puisque ses murs sont en pierre de taille ; les éléments d'ornementation en ciment sont gris pour les dallages et ocre pour les moulages ${ }^{4}-$ les ciments jaune de la Pérelle et rose d'Uriage n'existaient pas lors de la construction - et si ce monument de l'architecture néo-gothique du $\mathrm{XIX}^{c}$ siècle mérite quelque éloge, tout n'est pas du fait de son architecte (ill. 2).

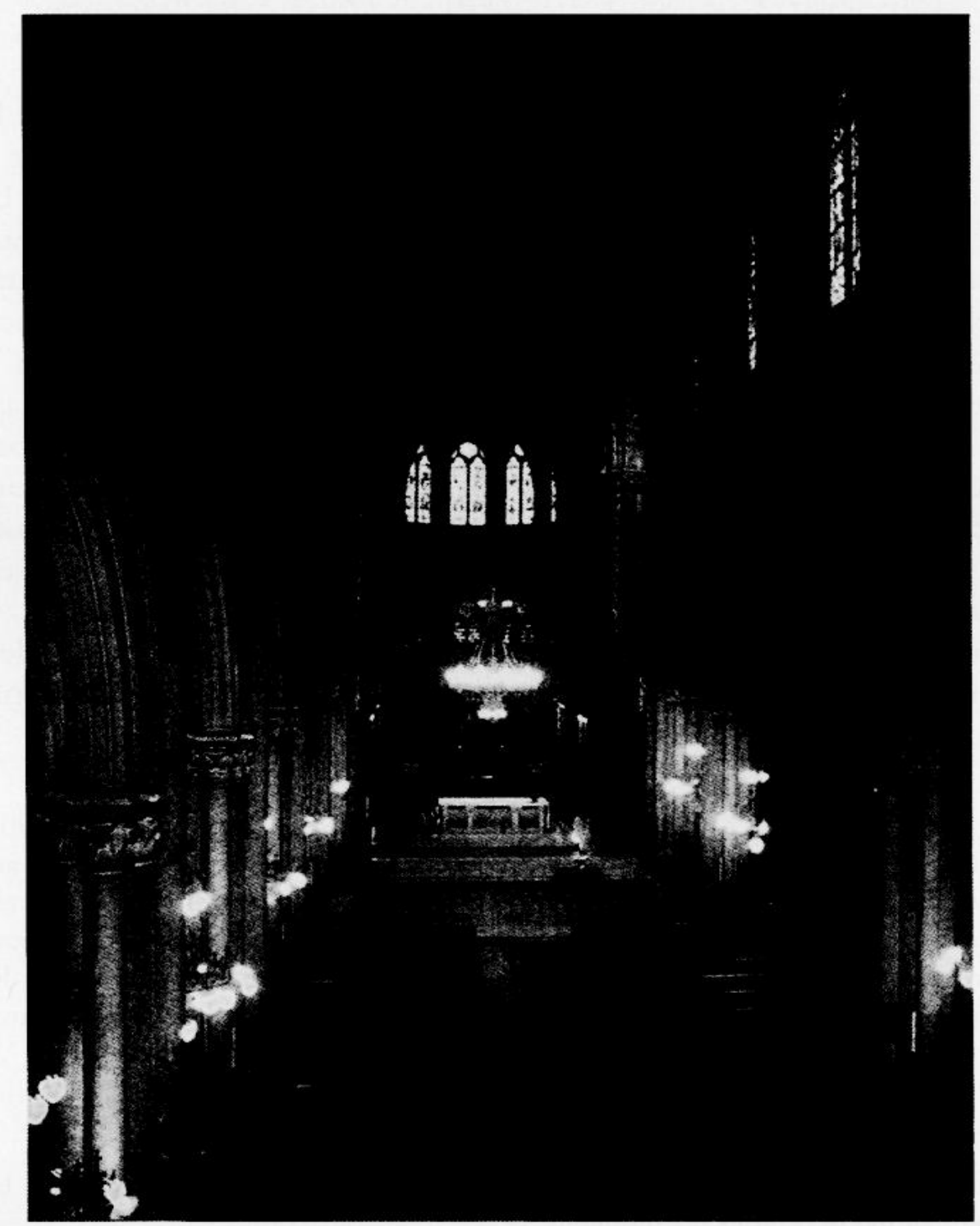

III. 2 : Alfred Berruyer, église Saint-Bruno de Voiron, nef principale (1857-1871). Carte postale.

4. Un ciment artificiel lent (portland) gris de Vicat et un ciment naturel prompt ocre de la Porte de France. 


\title{
Le rôle des protagonistes dans le style
}

Dès 1823, on se préoccupait de l'édification d'un nouveau lieu de culte dans le quartier des Terreaux, lieu insalubre à faire disparaître. Lorsque le maire Frédéric Faige-Blanc fut élu, en ami des Chartreux, il choisit l'architecte diocésain Alfred Berruyer qui, suivant les considérations de la commune, proposait dans son premier devis de 1857 : "Il faut faire une nouvelle église sur la place centrale, côté est, au-delà de la Morge, entre les deux ponts, en face de la grande fontaine. Cet édifice serait sujet à l'assainissement de l'endroit à son embellissement ${ }^{5}$. "On procéda rapidement aux expropriations de vingt-cinq propriétés pour un montant de 88898 francs $^{6}$. L'église devait être située au sommet de la grande place, chevet à l'est et façade à l'ouest donnant sur un boulevard.

Alfred Berruyer avait eu des projets concernant la reconstruction de la cathédrale de Grenoble : " La cathédrale est un monument trop modeste pour sa destination. Depuis longtemps, sa reconstruction aurait dû être entreprise ${ }^{7}$." L'État ne s'occupait pas de cette petite cathédrale et Berruyer ne recevait aucun soutien des administrations. Voiron, deuxième ville de l'Isère menée par un maire ambitieux, offrait la seule opportunité de bâtir un grand édifice gothique. Faige-Blanc comparait lui-même le chantier de Voiron à celui des cathédrales médiévales : "L'érection des grandes cathédrales, en même temps qu'elle fut une œuvre d'enthousiasme religieux, fut également une œuvre sociale et politique. [...] Dans ces vastes basiliques, enceintes civiles non moins que religieuses, les évêques et le clergé conviaient autour d'eux les cités populeuses et donnaient, par ces assemblées, son premier assaut à la féodalité ${ }^{8}$." De fait, la portée symbolique de Saint-Bruno ne fut pas seulement architecturale. Berruyer conçut un édifice simple, à trois nefs avec absides, un transept et deux tours, de $916 \mathrm{~m}^{2}$ pour 236000 francs avec 40000 francs de travaux supplémentaires à prévoir et 55000 francs pour les finitions à réaliser plus tard, mais la façade en constituait un élément majeur :

\begin{abstract}
La façade principale a trois portails antérieurs ornés de colonnettes, d'archivoltes et de tympans, la galerie à colonnettes arcaturées correspondant au triforium intérieur, les deux tours accusées par des contreforts puissants, percés de croisées géminées, avec soubassement pour les parties surélevées, la rosace de la grande nef, à double rang de colonnettes rayonnantes, le pignon, à rosaces murées, de la couverture de la grande nef avec la balustrade et les flèches des tours flanquées de contreforts ou tourelles évidées?.
\end{abstract}

5. Arch. mun. Voiron, $2 \mathrm{M} 3$, Alfred Berruyer, devis primitif pour la construction de la nouvelle église Saint-Bruno de Voiron, 25 novembre 1857.

6. 2 M 1, Mairie, acquisitions pour l'emplacement de l'église et d'une place, 1860-1873.

7. Alfred Berruyer, art. cit., p. 120.

8. Frédéric Faige-Blanc, Exposé au conseil municipal, n.d., cité dans François Crozet, Description topographique, bistorique et statistique des cantons formant le département de l'Isère et des communes qui en dépendent, Arrondissement de Grenoble, canton de Voiron, Grenoble, Prudhomme, 1869, p. 7.

9. 2 M 3, Alfred Berruyer, devis primitif pour l'église de Voiron, 25 novembre 1857. 
Berruyer commença ses plans en même temps que les souscriptions ${ }^{10}$. Le coût et la taille du monument ne permettaient pas de rentrer dans les critères de financement de l'État et la municipalité s'en passait. Sans que l'évêque eût laissé là-dessus de correspondance, sans qu'on n'eût parlé d'un éventuel desservant, la première pierre fut posée le 7 mars $1860^{11}$. Mais au mois de mai suivant, l'architecte devait, suivant l'avis du conseil des bâtiments civils présidé par Questel, simplifier son projet « à sa plus simple expression en vue de l'économie imposée par l'administration supérieure. [...] Il est question de ne monter qu'une grande masse sans ornements " ${ }^{12}$. Il parvenait à $1080 \mathrm{~m}^{2}$ pour 179000 francs en allongeant et en élargissant la nef. Il supprimait les arcs-boutants et faisait reposer la grande voûte directement sur les basses nefs. Les deux tours ne faisaient plus que 29 mètres de hauteur au lieu de 38 et les lanternes culminaient à 43 mètres contre des flèches prévues à 64 mètres. L'ornementation et les gargouilles avaient disparu. L'église n'avait plus son ossature gothique active et perdait une grande partie de son intérêt. Le conseil des bâtiments civils trouvait le projet mal chiffré : "Le prix au mètre carré est trop faible, 300 francs pour $1200 \mathrm{~m}^{2}$, au lieu de 500 francs à l'ordinaire " ${ }^{13}$ et demandait un troisième projet en décembre 1861 : "Il faut un projet plus simple en harmonie avec les ressources communales. Le style reste l'art ogival du XIII ${ }^{e}$ siècle, réduit à sa plus simple expression, surtout à l'extérieur. Tous les ornements ont disparu pour laisser la place toute entière à la pure construction. Des proportions ont été réduites. Il n'y a plus qu'un seul clocher placé contre le sanctuaire, ou sur la première travée ${ }^{14}$."

Un nouveau devis fut donc rédigé en décembre 1861 pour $950 \mathrm{~m}^{2}$ et 240000 francs bien que la commune ne pût emprunter que 210000 francs $^{15}$. L'État ne versa que $\mathbf{8 0 0 0}$ francs de secours au lieu des $\mathbf{4 0} 000$ francs demandés et le ministre de l'instruction publique et des cultes refusa "l'utilité publique " ${ }^{16}$. Les travaux furent ajournés en 1862 mais contre toute attente, le maire obtint du Conseil d'État une autorisation d'emprunt de 320000 francs en janvier $1863^{17}$. Les travaux des fondations et des soubassements furent adjugés en octobre 1863 et achevés en mai $1864^{18}$. Les travaux d'élévation allaient commencer mais le préfet refusa son approbation car on utilisait, sur une idée de l'architecte Berruyer, du ciment moulé au lieu de la pierre de taille, pour des raisons de coût ${ }^{19}$. Cette fois,

10. 2 M 6, grand livre des souscriptions de l'église Saint-Bruno de Voiron, 1857.

11. $2 \mathrm{M} \mathrm{2,} \mathrm{Frédéric} \mathrm{Faige-Blanc,} \mathrm{mairie} \mathrm{de} \mathrm{Voiron,} \mathrm{Pose} \mathrm{de} \mathrm{la} \mathrm{première} \mathrm{pierre} \mathrm{de} \mathrm{l'église} \mathrm{Saint-Bruno,}$ 7 mars 1860.

12. 2 M 3, Alfred Berruyer, devis pour l'église de Voiron, 10 mai 1860.

13. 2 M 2, Charles Questel, conseil général des bâtiments civils, Rapport, 29 octobre 1861. Nota: $1080 \mathrm{~m}^{2}$ pour 179000 francs font 165 francs $/ \mathrm{m}^{2}$.

14. 2 M 3, Alfred Berruyer, devis descriptif et estimatif de l'église Saint-Bruno de Voiron, 23 décembre 1861 .

15. 2 M 2, Mairie, extrait des délibérations du conseil municipal de Voiron, 18 janvier 1862.

16. 2 M 2, ministre de l'Instruction publique, lettre au préfet de l'Isère, 7 janvier 1863 .

17. 2 M 2, ministre de l'Instruction publique, lettre au préfet de l'Isère, 7 janvier 1863 .

18. 2 M 3, mairie de Voiron, extrait des délibérations du conseil municipal, 28 mai 1864.

19. 2 M 3, préfet de l'Isère, lettre au maire de Voiron, 10 juin 1864. 
la mairie passa outre et établit un contrat de gré à gré avec l'entrepreneur. Elle en profita pour modifier considérablement les plans autorisés et arranger un projet de 350000 francs. Berruyer laissa entendre que le projet primitif se montait à 550000 francs et qu'on obtenait donc une réduction de 200000 francs. Il expliquair encore: "Cette église exécutée en pierre de taille donnerait lieu à une dépense considérable. [...] Dès lors, la masse des édifices doit être en maçonnerie ou en bétons hydrauliques, avec un recouvrement léger en pierres tendres ou pierres factices. [...] L'église de Voiron, suivant les mêmes plans, avec les mêmes formes, exécutée en pierre de taille, comme on serait porté à le désirer ou à le croire, coûterait la somme de 1000000 francs $^{20}$. "Le maire était heureux de faire comprendre à ses contribuables et au conseil municipal que la commune allait se doter pour 350000 francs d'une église qui devrait valoir 1000000 francs en pierre - ce qui intéressa plus tard des hommes comme Louis Gillet, architecte de Châlonssur-Marne, ancien agent de l'entrepreneur en béton armé François Hennebique ${ }^{21}$. Le maire fit rajouter par l'entrepreneur de nombreuses ornementations en 1865 et demanda un devis supplémentaire d'un montant de 52509 francs $^{22}$. Il s'immisçait dans la maîtrise d'œuvre, faisait surélever le sol de deux assises, augmenter la longueur d'une travée, construire deux flèches en façade au lieu d'une à la croisée de transept, etc. Il demandait en somme à l'entrepreneur de suivre les plans du second projet de décembre 1863 , mais avec la façade monumentale du projet primitif de $1857^{23}$. Les travaux de toiture et de charpente furent adjugés dès le 8 avril $1866^{24}$, ceux du pont-parvis le 5 août $1866^{25}$, en même temps que la plâtrerie ${ }^{26}$, et ceux des voûtes, contreforts et sculptures des chapiteaux et colonnettes le 15 novembre suivant ${ }^{27}$. L'année 1867 vit apparaître les clochers, lanternes et plus tard les flèches ${ }^{28}$. Le montant réel des travaux s'élevait à 522000 francs au moins, sans la décoration peinte, le mobilier, l'orgue, ni les vitraux, le calorifere, etc.

Les relations s'envenimèrent en 1869 . Berruyer tenait à son église ${ }^{29}$ et le maire fit, seul, un projet d'ornementation soi-disant " approuvé par Viollet-le-Duc après une étude attentive des plans intérieurs " ${ }^{30}$, ce qui était faux. L'architecte répondit qu'il était " intolérable qu'il laiss[ât] réaliser des bas-reliefs sur le portail sans son accord " ${ }^{31}$. Le maire allait proposer à Berruyer " de démissionner si le préfet [était]

20. 2 M 3, Alfred Berruyer, rapport sur l'église Saint-Bruno de Voiron, 4 juin 1863.

21. Sylvain Mikus "Louis Gillet, architecte de la Belle Époque ", Mémoires de la Societé d'agriculture, commerce, sciences et arts du département de la Marne, Châlons-en-Champagne, 2001, p. 263-297.

22. 2 M 3, Alfred Berruyer, devis supplémentaire, 12 novembre 1865.

23. 2 M 2, Frédéric Faige-Blanc, mairie de Voiron; Ollivier-Pallud, entrepreneur, contrat, 10 juillet 1864 et extrait des délibérations du conseil municipal (adjudication de la deuxième partie de l'édifice comprenant la construction entière des murs), 10 juillet 1864.

24. 2 M 3, proces-verbal d'adjudication, 8 avril 1866.

25. $2 \mathrm{M} 3$, procès-verbal d'adjudication, 5 août 1866 .

26. $2 \mathrm{M} \mathrm{3}$, Alfred Berruyer, devis estimatif, 18 août 1866 .

27. $2 \mathrm{M}$ 2, extrait des délibérations du conseil municipal, 15 novembre 1866.

28. 2 M 2, extrait des délibérations du conseil municipal, 12 août 1867.

29. Il venait d'abandonner ses grands projets d'églises à Bourgoin et Saint-Laurent-du-Pont et de perdre son fils Flavien.

30. 2 M 2, Frédéric Faige-Blanc, lettre à l'architecte diocésain Alfred Berruyer, 5 mai 1869.

31. 2 M 2, Frédéric Faige-Blanc, lettre à l'architecte dioctsain Alfred Berruyer, 26 juin 1869. 
d'accord ${ }^{32}$, ce qui aurait suscité un vif conflit. Le préfet s'y opposa, estimant que "l'incompatibilité d'humeur " ne justifiait pas que le maire dût " se presser de faire ce qui lui plai[sait sur son œuvre " au risque d'entacher la "réputation " de l'architecte $^{33}$. En juillet 1869 , celui-ci démissionna de la direction du chantier.

Berruyer se battait pour l'intégrité de son cuvre mais dut abandonner l'ornementation au maire et les travaux d'achèvement à l'architecte Blandin. Il faillit ne pas percevoir ses honoraires, lesquels auraient dû être les plus importants de sa carrière, soit 18905 francs et ne les reçut qu'à la suite de longs procès en 1880 , dix ans après la réception définitive. Le coût total fut de 680000 francs : l'église paroissiale de Voiron avait été la plus coûteuse du diocèse. Le maire laissa donc entendre, pour se dédouaner, que les Chartreux avaient payé en grande partie la construction alors qu'ils limitèrent leur aide à 100000 francs destinés aux vitraux ${ }^{34}$. Cet édile ambitionnait une église majestueuse et croyait, convaincu par les lectures de son temps, que la majesté venait de la hauteur de l'édifice, la quantité des sculptures et l'importance des vitraux.

\section{Une " cathédrale " à Voiron?}

Les traits de l'église Saint-Bruno de Voiron sont ceux d'une cathédrale gothique, d'un gothique du XIII ${ }^{e}$ siècle idéalisé que l'on retrouve à l'intérieur dans la simplicité des volumes et l'élan des colonnes et à l'extérieur dans la perfection formelle de la façade principale, symétrique, aux éléments sobres et strictement verticaux. La couleur des peintures de l'intérieur et de l'ornementation ocre sur la pierre blanche de l'extérieur corrigent la sécheresse mathématique. Saint-Bruno répond à l'idée abrégée que Berruyer se faisait du gothique : "Les traits caractéristiques sont l'arc surélevé à ogive, l'élancement des colonnes en vue de l'exhaussement général de l'édifice, les voûtes à nervures à ogives portées par des pieds-droits réduits à la place des murs, la prédominance des lignes verticales sur les horizontales, les plans symboliques qui affectent la forme du Christ en croix " 35 .

Le plan au sol est excessivement simple, une croix latine peu marquée sans chapelles ni déambulatoire. L'élévation utilise un système presque passif et trop massif pour se rapprocher du gothique rationaliste. Les tours et les fleches font largement défaut. Quant à la façade, elle est bien trop plane pour provenir de la cathédrale idéale de Viollet-le-Duc ou de son modèle Notre-Dame de Reims, aux nombreux décrochements. Du reste, la "cathédrale idéale" fut publiée en 1865 seulement, lors de la parution du deuxième volume du Dictionnaire raisonné d'architecture ${ }^{36}$, c'est-à-dire sept ans après les plans de Berruyer ${ }^{37}$.

32. 2 M 2, Frédéric Faige-Blanc, lettre à l'architecte diocésain Alfred Berruyer, 3 juillet 1869.

33. 2 M 2, Alfred Berruyer, lettre au maire de Voiron M. Faige-Blanc, 5 juillet 1869.

34. 2 M 2, Charles Marie, prieur de la Grande Chartreuse, lettre au Maire de Voiron, $1^{\text {er }}$ avril 1863.

35. Alfred Berruyer, " Discours de réception ... ", art. cit., p. 107.

36. Eugène-Emmanuel Viollet-le-Duc, Dictionnaire raisonné de l'architecture franfaise du $X I^{e}$ au $X V T^{*}$ siecles, Paris, 1865 , t. II, p. 324, art. * cathedrale ".

37. Les séries de plans de Berruyer sont datées respectivement du 25 novembre 1857, 10 mai 1860 , 23 décembre 1861 et 4 juin 1863 (2 M 3). 
La façade de Saint-Bruno se rapproche au contraire de Notre-Dame de Paris et de la composition théorique de la cathédrale idéale du $\mathrm{XIX}^{\mathrm{e}}$ siècle $^{38}$. Elle cède à une tripartition horizontale et verticale sur un schéma presque carré : trois portails à la base, puis une rosace et deux baies au milieu et, enfin, les bases des deux tours et le pignon du grand comble, formant ainsi une grille de lecture logique. Les ouvertures annoncent les divisions intérieures de l'édifice. Chacun des trois portails renvoie à un vaisseau et chacun des trois niveaux à un étage. Le portail central et la rosace attestent de la largeur et de la hauteur de la nef principale. Les portails des bas-côtés surmontés d'une galerie d'arcades et de fenêtres géminées annoncent l'élévation intérieure. C'est ce qu'avait recommandé Berruyer lui-même en 1857, en particulier pour ce qui concerne la galerie d'arcades inférieure à placer à la hauteur du triforium ${ }^{39}$. Ces dispositions en font une véritable façade harmonique, une petite Notre-Dame dont les flèches auraient été achevées.

Le corps de l'église Saint-Bruno suit un plan commun avec une élévation néogothique simple, presque épaisse, avec des moulures éparses en manière d'ornementation mais sa façade est un chef-d'œuvre d'harmonie, le seul élément d'architecture du département soumis aux règles du gothique archéologique pour laquelle Berruyer s'est plus qu'inspiré du nouveau Sacré-Cœur de Moulins que Jean-Baptiste Lassus et Louis-Gabriel Esmonnot avaient commencé en 1849.

Alors que la peinture religieuse traversait, en général, une sorte de renaissance, en Isère, on ne connut guère autre chose que des travaux de badigeonneurs à part quelques compositions dues à Alexandre Debelle $(1805-1897)^{40}$ et une peinture de Dominique-Alexandre Denuelle (1818-1879) à la cathédrale de Grenoble. Le projet de décoration de l'église Saint-Bruno de Voiron, tâche primordiale pour le commanditaire convaincu qu'aucune tradition décorative n'égalait le $\mathrm{XIII}^{e}$ siècle et qui signait son projet "Cartusia, nunquam reformata, qui[a] nunquam deformata", paraît alors fondamental. La commission chargée de la décoration explique pourtant dans son livret édité spécialement qu'il ne s'agit pas d'entreprendre l'exécution d'un pastiche malheureux, mais de rechercher " par l'étude des XII et XIII" siècles, les éléments d'une peinture décorative sobre et discrète " ${ }^{41}$. Ayant pris connaissance des travaux de Jacques-Ignace Hittorff à Saint-Vincent-de-Paul (Paris), cette commission, la mieux documentée du département, voyait dans la coloration brillante " une auxiliaire de la sculpture d'ornementation " et expliquait : " L'architecture est avant tout un art de raison. Non seulement elle exige la solidité, mais elle veut

38. Voir "La cathédrale idéale", dans Jean-Michel Leniaud, Les Cathédrales au XIX siècle, op. cit., p. 503-505.

39. Berruyer avait aussi dessiné en 1857 une variante de la façade dont la galerie d'arcades supérieure passait devant le pignon à la manière de la balustrade de Notre-Dame de Paris.

40. Voir Sylvie Vincent, "L'art du religieux" et "Reconstructions et ornementations des églises au XIX siècle en Isère ", Alexandre Debelle, peintre dauphinois, Grenoble, C.P.I., éd. Cent pages, à parâtre.

41. Frédéric Faige-Blanc, maire et président de la commission municipale chargée de la surveillance des travaux, Projet de decoration interieure de l'eglise Saint-Bruno de Voiron, Grenoble, Allier père et fils, 1868 , p. 15 et suiv. 
encore l'apparence de la solidité." C'est pourquoi il fallait choisir une " peinture discrète et néanmoins savante " parmi les sept ou huit en France dont le meilleur exemple se trouvait dans le cloître des Jacobins d'Agen qui fut copié.

Face à la simplicité des peintures, le chantier exceptionnel de Saint-Bruno de Voiron devait recevoir des vitraux de la grande maison Laurent Gsell de Paris, réalisés par M. Ruin dès 1869 pour 100000 francs. Frédéric Faige-Blanc, qui, possédant un nom de plume, Alpinus, se croyait critique d'art et invoquait le passé en matière de décoration ${ }^{42}$, expliquait son aspiration pour les grandes cathédrales du Moyen Âge et la forme des basiliques, le triforium des grandes églises du nord, la toiture polychrome de Saint-Bénigne de Dijon et les grandes verrières de Chartres, Bourges ou Poitiers encore qu'elles fussent " incorrectement dessinées ». La couleur devait primer sur les tableaux, contrairement aux idées des ecclésiastiques, et cela le rendait sûr de lui car, disait-il : "Nous avons puisé les éléments dans les publications de l'Académie des sciences et de la commission des monuments historiques, dans les instructions du comité des Beaux-Arts et dans les œuvres critiques ou autres de MM. Didron, Viollet-le-Duc, Mérimée, César Daly, Lassus, Chevreul, etc. $"$ " 3 .

Frédéric Faige-Blanc passa une commande originale, finalement la seule de son église qui ne fût copiée sur aucun chef-d'œuvre, concernant le thème de la charité : le père nourricier. L'homme est vêtu de bure avec une corde au cou et un grand cierge, suivi de deux clergeons et de la population. On le retrouve accompagnant l'évêque de Grenoble en visite auprès des malades de la peste de 1628-1630 à Voiron, posant la première pierre de la nouvelle église ou offrant l'édifice aux Chartreux. Le personnage n'est pas nimbé car ce n'est pas un saint. C'est un homme du pays, lui ${ }^{44}$.

\section{Un palimpseste néo-roman : l'église Saint-Bruno de Grenoble (1865-1881)}

Après la visite de Napoléon III en $1860^{45}$, la ville de Grenoble s'est décidée à se doter d'attributs magistraux en commandant à Charles Questel la restauration du palais de justice, la construction d'un palais de préfecture et d'un muséebibliothèque sur la place d'armes (actuelle place de Verdun), construits de 1861 à 1872. À cela s'ajoutaient l'hôtel des facultés et le siège de la division. Une politique de construction germait enfin ${ }^{46}$. Le plan d'extension de la ville allait vers l'ouest,

42. Pour reprendre Salvatore Setris, L'Invention d'un tableau "La tempête de Giorgione ", Paris, éd. de Minuit, p. 11.

43. Frédéric Faige-Blanc, Projet de décoration intérieure de l'église Saint-Bruno de Voiron, op. cit., p. 15 et suiv.

44. Se faire représenter de son vivant dans une église était interdit par la Sacrée Congrégation des rites car le geste était perçu comme une preuve de piété orgueilleuse.

45. Victor Advielle, L'Empereur Napoléon III à Grenoble et dans le département de l'Isère les 5, 6 et 7 septembre 1860, Grenoble, Merle. A, 1860, 47 p.

46. Emile Vaudremer construisit le grand lycée de garçons (lycée Champollion) en 1883. 


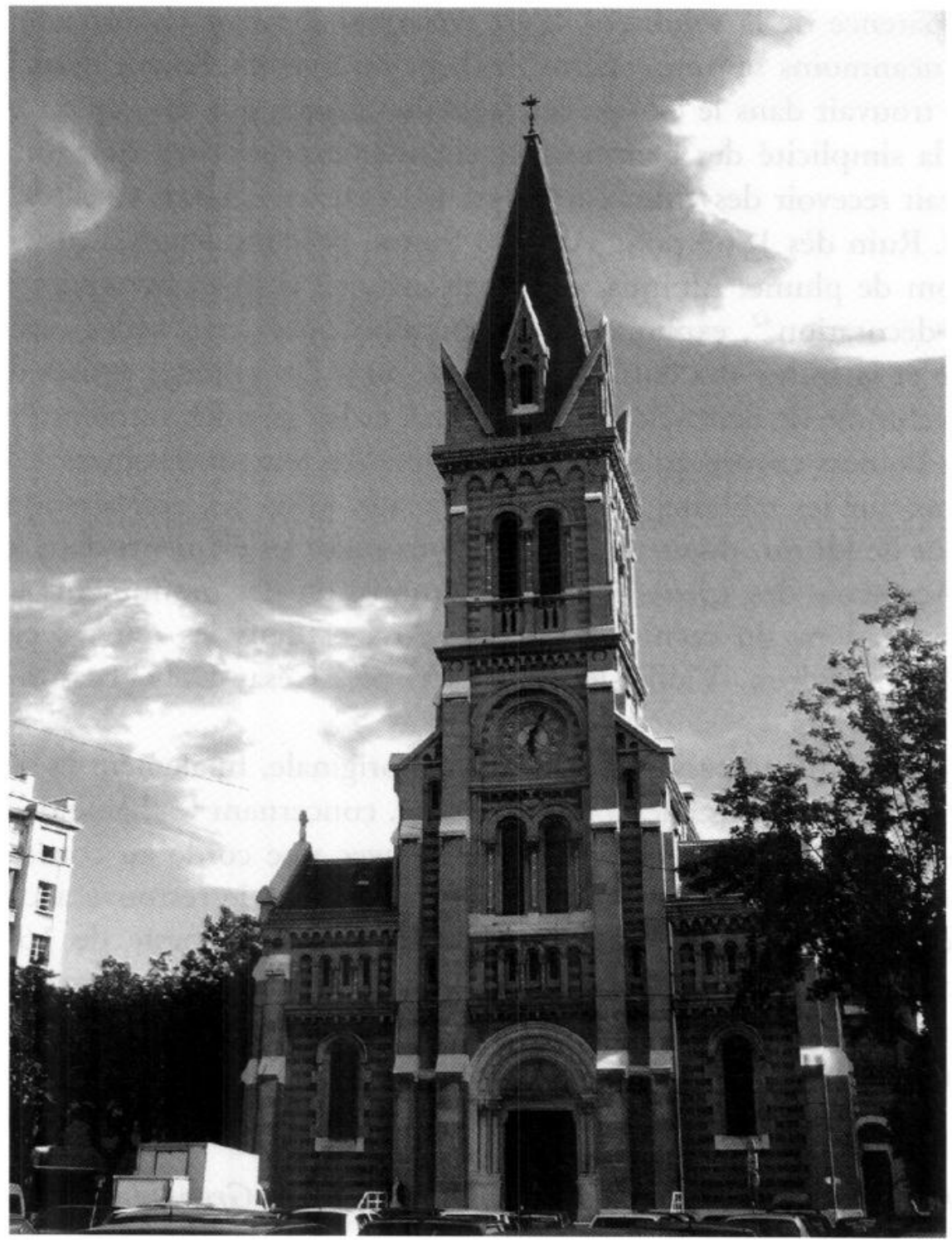

Ill. 3 : Alphonse Durand, Eugène Péronnet, Alfred Berruyer, église Saint-Bruno de Grenoble, 18691881, façade principale. Cl. C. Avenier.

englobant la plaine de Montrigaud qui allait devenir le nouveau quartier SaintBruno dont la grande église Saint-Bruno serait l'objet d'un concours international et fut élevée rapidement, entièrement en ciment moulé (ill. 3). De fait, la population fit de l'architecte qui opéra la réception d'œuvre, le diocésain Alfred Berruyer, célébrité qui venait d'achever l'église de Voiron, un champion de l'architecture, " un rénovateur de l'art, contre les églises de la Restauration [...] contre le style grange $"{ }^{47}$. Il fut d'autant héroïsé qu'il ne s'entendait pas avec la municipalité anticléricale d'Édouard Rey qui refusa de le remercier dans son discours prononcé lors de la bénédiction. Berruyer aurait dessiné un grand édifice néo-roman, financé par

47. Bibl. mun. Grenoble, Pd (1) 1, anonyme, "Alfred Berruyer, auteur des plus belles églises du diocèsc de Grenoble ", Almanach dauphinois, Grenoble, 1903, 1 p. 
les Chartreux, à la gloire du ciment, qui a servi de modèle au Siam et à Madagascar et dont la décoration picturale n'a pas été réalisée. Il n'est pas même l'auteur du projet pour lequel aucune peinture n'a jamais été prévue.

\section{Un concours joué d'avance?}

En 1865, la ville de Grenoble se divisait en quatre paroisses intra-muros et une paroisse extra-muros, Saint-Joseph qui, avec l'exode rural et l'urbanisation, devint très rapidement la paroisse la plus importante ${ }^{48}$. Mgr Achille Ginoulhiac caractérisait ainsi les paroissiens de la périphérie travaillant dans les nouvelles usines de la section de Montrigaud : " une population dont la majeure partie est ouvrière, pauvre, flottante, et a besoin plus que toute autre d'un centre religieux " ${ }^{49}$ et décida de transformer la chapelle Saint-Bruno en succursale en 1866, puis en cure indépendante en $1872^{50}$. L'emplacement de l'église fut une cause de discordes pendant plusieurs années ${ }^{51}$ et la ville dut constituer une commission spéciale en 1872 qui proposait cinq endroits possibles ${ }^{52}$, notamment devant le marché aux légumes, à côté du marché aux bestiaux, sur la ligne de chemin de fer de Chambéry, etc. L'édifice fut finalement installé au sud de la place et la façade au nord en fonction des boulevards alentours ${ }^{53}$. Initialement, la ville de Grenoble ne souhaitait pas de concours car « l'architecte choisi pourrait être un élève d'une grande école mais inexpérimenté à conduire des travaux, ce qui engendrerait des dépenses non prévues ». À Grenoble, on se méfiait des parisiens et de leur luxe : "On s'est adressé à Paris, aux sommités artistiques. On crut arriver ainsi à la perfection. Les résultats en sont une dépense énormément augmentée et le côté artistique des monuments prête largement à la critique $"^{54}$. Une partie du conseil municipal refusait aussi ce projet car la ville prévoyait depuis longtemps d'élever une grande église dans le vieux centre. Hector Riondel (1833-1903), architecte départemental, faisait donc un premier projet pour servir de base de travail aux soumissions des architectes futurs candidats : "L'église Saint-Bruno ne doit pas être un monument dispendieux et plus important que ne le comportent les besoins du quartier. [...] Il faut laisser le plus de place possible au marché aux bestiaux et devant l'église. Il ne faut pas faire de porche, ce qui est trop cher. L'église doit avoir une grande nef de 9,8 $\mathrm{m}$ de large divisée en sept travées terminée par une abside carrée plus écono-

48. Arch. dép. Isère, $3 \mathrm{~V} 3$, évêché, circonscription des paroisses de Grenoble, plan, population, 3 mai 1845.

49. Arch. mun. Grenoble, $2 \mathrm{M} 26$, pétition des habitants du quartier Saint-Bruno de Grenoble, 2 novembre 1866.

50. Arch. dép. Isère, 3 V 3, ministère de l'Instruction publique et des Cultes, décrets, 2 juin 1866 et 20 novembre 1872.

51. Arch. mun. Grenoble, 2 M 26, préfet Pastoureau, Arrêté pour une enquête, 4 janvier 1866.

52. Bibl. mun. Grenoble, $O$ 16655, commission des travaux, emplacement de l'glise Saint-Bruno, 23 seprembre 1872.

53. Arch. dép. Isère, 2 M 26, préfet de l'Isère, arrêté pour l'acquisition de terrains, 10 juin 1873.

54. $2 \mathrm{M}$ 29, Jean-Thomas Vendre, maire, extrait des registres des délibérations du conseil municipal de Grenoble, 19 juin 1869. 
mique $^{55}$. "Ce projet d'église à 300000 francs reçut l'appui du département et du nouveau curé, l'abbé Martin Berlioux qui, à cause de ses élans bâtisseurs, fut un des rares religieux à faire l'objet de violentes critiques anticléricales ${ }^{56}$. L'abbé reçut très rapidement, en 1868, le soutien des Chartreux : " 100000 francs, payables en cinq annuités de 20000 francs à commencer de l'an 1870 " ${ }^{57}$. Il aurait aussi trouvé des fonds supplémentaires chez la reine Victoria qu'il avait sollicitée alors qu'elle se trouvait dans son train pour Aix-les-Bains. De son wagon stationné à Grenoble, elle pouvait observer le quartier en construction et le terrain vide qui devait accueillir l'église. Lorsqu'elle donna, le curé resta là et se permit de redemander. Il le fit cette fois à l'Impératrice des Indes. Son Altesse apprécia le double titre qui lui était rarement concédé, et augmenta sa première libéralité ${ }^{58}$.

De garde-fou en garde-fou, la commune demanda un second projet en $1869^{59}$, puis on finit par se résoudre à organiser le premier concours d'architecture en Isère. L'édifice servirait à mettre en valeur les nouveaux quartiers de l'ouest et on laisserait en paix les "vieilles églises". Le 17 mai 1874, la première pierre fut posée, en présence de nombreuses personnalités mais sans les Chartreux car "la place des enfants de saint Bruno est dans la solitude plutôt qu'au milieu des solennités. L'église fut bénite le 15 septembre $1878^{60}$ et les travaux soldés en 1881. En quinze années, l'abbé Berlioux fit de Saint-Bruno une grande paroisse avec le plus haut, 72 mètres, monument de Grenoble.

Le concours était international, le programme officiel de la Ville était volontairement imprécis : "Aucun style spécial n'est imposé mais la dépense totale de l'église, ameublement et honoraires ne dépassent pas 300000 francs " ${ }^{61}$ pour un édifice de $900 \mathrm{~m}^{2}$. En 1867, pourtant, "sur le désir manifeste de plusieurs membres du clergé, [la fabrique avait] demandé que le projet [fût] conçu dans le style du XIII' siècle " ${ }^{62}$. Il fut envoyé en août 1869 à cent soixante personnes dans trente-sept villes différentes et annoncé par voie de presse, dans les journaux régionaux et parisiens, dans quelques revues d'architecture ${ }^{63}$. Le travail portait ses fruits car soixante-sept projets sont enregistrés dès la fin de l'année ${ }^{64}$. Des noms impor-

55. 2 M 27, Hector Riondel, premier projet de construction de l'église Saint-Bruno, 1867.

56. 4 V 59, anonyme, couplets contre M. Berlioux, curé de Saint-Bruno de Grenoble, envoyés du commissariat central de Grenoble au préfet de l'Isère, le 6 mai 1867 : « un excellent giboyeur, en état de paternité [...] et tonton-tonton les saints polissons (bis). "

57. Frère Charles Marie, prieur de Chartreuse, lettre au Maire de Grenoble, 22 octobre 1868.

58. Emile Romanet, Quelques pages d'bistoire paroissiale. Saint-Bruno et ses auvres, Grenoble, Imp. Saint-Bruno, 1941, p. 2

59. 2 M 27, Hector Riondel, deuxième projet de construction de l'église Saint-Bruno, 1869.

60. Elle n'a pas été consacrée.

61. Ville de Grenoble, Construction d'une église sous le vocable de Saint-Bruno, dans la commune de Grenoble extra-muros. Programme du concours. Bordereau de prix, Grenoble, Dardelet, 1869, 26 juillet 1869 , p. 1.

62. 2 M 27, Hector Riondel, premier projet de construction de l'église Saint-Bruno, 1867.

63. 2 M 29, Eugène Péronnet, liste des destinataires du programme pour le concours de la construction de l'église Saint-Bruno, 10 août 1869.

64. 2 M 29, mairie de Grenoble, enregistrements des projets du concours de Saint-Bruno, 31 décembre 1869. 
tants répondaient, comme Edouard Corroyer, Louis-Jean Sainte-Marie Perrin ${ }^{65}$. Á Grenoble, il pouvait intéresser trois architectes : Alfred Berruyer, Eugène Péronnet (1807-1877), auteur de nombreuses églises et cousin germain de Félix Breton, le directeur des Ciments de La Porte de France et premier adjoint à la mairie, et Hector Riondel, architecte départemental et futur neveu par alliance de Péronnet. Le jury était composé entre autres personnes de trois architectes : Charles Questel, alors président du conseil des bâtiments civils à Paris, son inspecteur départemental Hector Riondel et Eugène Péronnet. Trois architectes furent primés en 1870 : Anatole de Baudot (1834-1915), Jean Litoux (1839-1903) et Alphonse Durand (1814-1882). Alfred Berruyer ne prit pas la peine de concourir puisque ses adversaires éternels Péronnet et Riondel étaient membres du jury.

Anatole de Baudot reçut le premier prix et pensait sérieusement élever son monument (ill. 4). On avait peut-être apprécié en lui le successeur de Viollet-le-

Ill. 4 : Anatole de Baudot, Projet pour l'église Saint-Bruno de Grenoble, 9 juillet 1870, premier prix au concours. Archives municipales de Grenoble, 2 Fi 975.

65. Croquis d'architecture Intime Club, $n^{\circ}$ XII, f. 6, avril 1870 et $n^{\circ}$ I, f. 6, mai 1870. 
Duc et l'auteur d'Églises de bourgs et de villages ${ }^{66}$, livre de modèles d'églises édité en 1867 et destiné à aider les architectes et les maîtres d'ouvrage. Son projet, sans titre, offrait un parti romano-gothique plutôt simple. L'église serait érigée sur une place. Il pouvait donc développer un plan en croix latine, composé de trois nefs et d'un chevet circulaire augmenté pour accueillir les stalles. Les ouvertures de passages et les voûtements avaient un profil de berceau brisé. Les ouvertures supérieures étaient romanes. La façade principale, tripartite et pyramidante, était surmontée d'une rose et d'un clocher-porche dotée d'une flèche élancée : un projet presque commun, composé d'un rappel gothique, car la fabrique l'avait demandé, et d'éléments romans. Finalement, il reprenait presque exactement l'allure des avants-projets de Riondel qui, lui, s'était inspiré de la cathédrale de Gap par Charles Laisné à l'exception des tours-escaliers ${ }^{67}$. Baudot ne pouvait se contenter de petites ouvertures ogivales, ni pour les bas-côtés ni pour les fenêtres hautes. Il prenait des roses à six lobes qu'il faisait surmonter d'arcs lombards pour la nef centrale, conservait les petites ogives des bas-côtés mais les encadrait à chaque travée par un arc ogival. Les ouvertures des portails prenaient de l'ampleur pour devenir des baies triples. Le chœur, non destiné à être vu depuis que la Ville souhaita reculer l'église, rapetissait ainsi que les ouvertures. Le clocher, désormais sur la place du grand marché, prenait quant à lui de la hauteur; Baudot se rapprochait de la cathédrale de Gap qu'il dotait de la flèche de l'église de Valeyrac (Gironde), conçue par Paul Abadie et publiée dans son recueil. Ce projet offrait un caractère régional légèrement italianisant.

Le second prix alla au projet présenté sous la signature Yan. L'auteur en était Jean Litoux, élève de Questel. Il avait été jugé trop grand en taille, ce que montrent, en effet, les plans. L'édifice n'était pas élancé mais particulièrement vaste, surmonté de coupoles sur plan circulaire tant dans la nef que dans le transept. Les ouvertures étaient en plein cintre et le profil des coupoles repris en dessins sur les murs extérieurs des bas-côtés. L'aspect romano-byzantin du corps de l'édifice est clair mais jure avec le clocher-porche doté d'une seule ouverture et flanqué de deux tours octogonales.

Le troisième prix, celui d'Alphonse Durand (ill. 5), ancien diocésain d'Autun et de Coutances, inspecteur des travaux de la Ville de Paris, portait la devise Et $U_{n u s^{68}}$. Le plan était simple, suffisamment pour convenir au mieux : une croix latine, une vaste croisée de transept, un clocher porche ouvrant à gauche et à droite sur les fonts baptismaux et une chapelle des morts, deux sacristies situées de chaque

66. Anatole de Baudot, Eglises de bourgs et de villages, Paris, Morel, A., 1867, 2 vol. n.p.

67. Riondel s'inspirait de Charles Laisné car le modèle de la cathédrale de Gap se prêtait à la ville de Grenoble mais Riondel s'attirait aussi les sympathies de Laisné qui venait, en 1869, d'être nommé en remplacement de Pierre Manguin comme directeur des travaux de restauration de l'église SaintLaurent, monument historique important de Grenoble.

68. Ce projet n'est pas signé et le titre du projet de Durand n'est pas connu mais il est très proche de l'avant-projet de Riondel du projet de Baudot et surtout de l'église actuelle. Sachant que Péronnet et Berruyer ont apporté chacun des modifications, ce rapprochement Durand/Et Unus, est possible. Enfin, il correspond à la critique qu'en a faite Questel en 1873 en tant que rapporteur au conseil général des bâtiments civils. 
Ill. 5: Alphonse Durand, Projet pour l'église Saint-Bruno de Grenoble, Et Unus, troisième prix au concours (choisi pour la construction), 1870. Archives municipales de Grenoble, 2 Fi 963.

côté du sanctuaire accessibles par le dehors et par les bras de transept. L'édifice paraissait un peu petit, $52 \mathrm{~m}$ de longueur et 23 de largeur qui faisaient $877 \mathrm{~m}^{2}$ de surface utile, mais facile à agrandir par l'élargissement des collatéraux. L'élément le plus intéressant, "méritant " pour reprendre Questel, était le " caractère de simplicité et de fermeté " de l'ensemble et, particulièrement, de la façade. En revanche, les éléments ornementaux furent critiqués : "Les murs pignon ne devraient pas être coupés horizontalement par l'espèce de bandeau avec corbeaux qui divise le mur dans sa hauteur ; pareil pour les murs du chevet. Les fenêtres de la grande nef nous paraissent avoir une importance trop considérable ${ }^{69}$."

69. 2 M 29, Charles Questel, ministère des travaux publics, rapport du conseil général des bâtiments civils, 21 novembre 1873 . 
Le premier prix dépassait largement les limites budgétaires et Baudot fut incapable de le corriger selon les souhaits municipaux de 1872, refusant systématiquement le ciment moulé. La ville se reporta le 15 octobre 1873 sur le troisième prix d'Alphonse Durand ${ }^{70}$. Le 18 octobre, il se mettait à la disposition du maire de Grenoble lorsqu'on lui apprit qu'Eugène Péronnet rédigeait déjà une série de prix avec le jeune Jules Riondet $(1847-1887)^{71}$. La Ville avait choisi de lancer un concours afin de se procurer des modèles à réaliser par un architecte local. Riondel avait établi les projets primitifs et Péronnet obtint la direction du chantier. Questel et la commission voulaient de la simplicité et de l'ampleur, soit du caractère mais pas de dépenses. Péronnet s'employait donc à réécrire un devis estimatif. Il élargissait la nef, supprimait quatre pinacles au clocher ainsi que les bandeaux horizontaux, agrandissait les fenêtres, ajoutait des moulures " avec jets d'eau " du Moyen Âge, renforçait les contreforts ${ }^{72}$.

Les desiderata du maître d'ouvrage avaient bridé l'imagination avant le concours. Les avants-projets de Riondel, presque des projets plagiaires ${ }^{73}$, amenèrent les participants plus à copier qu'à inventer. Peut-être avaient-ils eux-mêmes suivi ce chemin peu créatif afin de remporter un concours dont ils n'espéraient de toutes façons pas pouvoir réaliser une œuvre extraordinaire puisque la commission des bâtiments civils imposait toujours des simplifications.

\section{Un chantier approximatif}

En 1873, avant même le début des travaux, une commission municipale fut chargée de surveiller le chantier; les rapporteurs s'appelaient Finet et Gauthier ${ }^{74}$. Les entrepreneurs offraient des rabais considérables pour obtenir cette construction prestigieuse. Joseph Serpolet, mouleur en ciment, fut adjudicataire après avoir concédé 17,5\% de rabais. De 1873 à 1875, l'entrepreneur Serpolet avait bâti les fondations, le soubassement et commençait l'élévation des premières assises. Cette année 1875, l'architecte Péronnet devait diriger l'élévation. Il s'appuyait sur des plans et devis confus. Les plans approuvés donnaient des colonnes en plusieurs fâts et une flèche en charpente alors que le devis approuvé mentionnait des colonnes monolithes et une flèche en ciment. Serpolet n'arrivait plus à s'organiser. Il fit quatorze réclamations le 27 janvier 1875 et demanda la résiliation de son marché le

70. 2 M 29, F. Finet, rapport fait au nom de la commission des travaux sur le projet de construction de l'église Saint-Bruno, 15 octobre 1873.

71. 2 M 29, Alphonse Durand, lettre au maire de Grenoble (contenant une réponse), 18 octobre 1873.

72. 2 M 29, Eugène Péronnet, modifications apportées à l'́glise Saint-Bruno à la suite de celles demandées par le conseil général des bâtiments civils, lettre envoyée au maire de Grenoble, 29 décembre 1873.

73. Toutes les églises de Riondel ont été copiées dans le livre d'Anatole de Baudot Eglises de bourgs et de villages dont celle de Saint-Sauveur (Pyrénées-Orientales) par Émile Boeswillwald pour Eybens et celle d'Aillant-sur-Tholon (Yonne) de Viollet-le-Duc pour Izeaux; il adoptait ici le beffroi et la flèche de l'eglise de Masny (Nord), par Boeswillwald.

74. 2 M 29, F. Finet, au nom de la commission des travaux sur le projet de construction de l'église Saint-Bruno, rapport, 15 octobre 1873. 
4 février car il faisait faillite. Péronnet écrit au maire qu'il corrigerait ces incohérences en apportant des modifications ${ }^{75}$. En mars 1875 , la commission envoya une délégation sur le chantier qui fit ces constatations :

" $1^{\circ} \mathrm{La}$ porte principale qui doit être pourvue d'un pilier la divisant en deux et un tympan plein cintre, est remplacée par une baie plus étroite, plus haute et sans trumeau central surmontée d'un cintre surhaussé qu'un fronton triangulaire termine au sommet.

$2^{\circ}$ Des piliers rectangulaires sont substitués aux colonnes.

$3^{\circ}$ Les sacristies agrandies débordent du transept jusqu'à faire une masse unique $^{76}$."

Les différends entre l'architecte et l'entrepreneur étaient si importants que la ville créa une seconde commission indépendante dirigée par l'architecte Tony Desjardins, diocésain de Lyon, qui choisit Louis-Maurice-Antoine Bresson, membre du conseil des bâtiments civils du Rhône et son collaborateur, pour le seconder ${ }^{77}$. Il ensuivit " une grande responsabilité morale à l'architecte, celle d'avoir apporté à une œuvre conçue avec sagesse et gravité un certain trouble qui nuit à l'ensemble du monument sans que le surplus d'ornementation qui a été introduit lui soit en rien profitable, tant au point de vue décoratif qu'à celui d'une plus grande solidite ${ }^{78} "$.

Péronnet prit sa retraite cette année et Serpolet fut résilié. Péronnet avait souhaité que la commune le remplaçât par Émile Chatrousse (né en 1847) et Joseph Ricoud (1850-1903), ses successeurs ${ }^{79}$. Jules Riondet, son assistant, avait de son côté aussi sollicité la direction du chantier ${ }^{80}$. Mais la commission ne fut pas satisfaite, d'autant que Chatrousse, Ricoud et Riondet avaient tous les trois entre vingt-cinq et vingt-sept ans. Elle choisit Berruyer " qui paraissait avoir beaucoup d'expérience et un talent attesté par de nombreux édifices construits " ${ }^{81}$. Desjardins remplaça donc Péronnet par Berruyer, très expérimenté certes mais aussi son homologue et ancien assistant à Lyon, et Berruyer remplaça Serpolet par la société Rayneri frères qu'il connaissait déjà ${ }^{82}$.

La mission du nouvel architecte et maître d'œuvre consistait à revenir aux formes du projet précédemment approuvé tout en ménageant la transition avec la partie déjà exécutée. Il transforma néanmoins la façade principale ${ }^{83}$, sans placer le

75. 2 M 31, Eugène Péronnet, lettre au maire de Grenoble, 15 février 1875.

76. 2 M 30, F. Gauthier, commission des travaux et du contentieux, (Ernest Calvat, maire, Jules Flandrin, Eugène Charpenay, Diodore Rahoult, Auguste Gaché, etc.), rapport envoyé au conseil municipal de Grenoble, $1^{\text {er }}$ mars 1875.

77. 2 M 31, Tony Desjardins, lettre au maire de Grenoble, 22 mars 1875.

78. 2 M 31, Tony Desjardins, architecte diocésain de Lyon ; Louis Bresson, membre du conseil des bâtiments civils du Rhône, rapport rendu à la mairie de Grenoble, 7 mai 1875.

79. 2 M 31, Eugène Péronnet, lettre au maire de Grenoble, 24 juillet 1875.

80. 2 M 31, Jules Riondet, lettre aux adjoints de la ville de Grenoble, 28 juillet 1875.

81. 2 M 31, ville de Grenoble, extrait du registre de deliberation du conseil municipal, 2 août 1875.

82. 2 M 31, Tony Desjardins; Louis Bresson, rapport, 7 mai 1875.

83. 2 M 30, Alfred Berruyer, lettre a Auguste Gaché, maire de Grenoble, 30 mai 1876. 
trumeau prévu et en demandant au sculpteur Henri Ding de réaliser les quatre chapiteaux du portail en forme des quatre évangélistes ${ }^{84}$. Berruyer affichait encore quelques prétentions pour l'église : des piédroits (piliers fasciculés) avec colonnettes ${ }^{85}$, une flèche en bois couverte d'ardoises ou mieux de feuilles de cuivre et des arcs-boutants à exécuter en régie ${ }^{86}$. Les arcs-boutants furent posés sous les combles des bas-côtés, à la manière de Viollet-le-Duc et comme à l'église SaintBruno de Voiron. Le conseil des bâtiments civils refusait la feuille de cuivre comme un luxe, et ridicule du point de vue stylistique, mais autorisait l'ardoise ${ }^{87}$.

Alfred Berruyer utilisa également des éléments de son répertoire personnel : les colonnettes roses réservées aux galeries d'arcades; les modillons à doucine renversée inspirés de l'antique et disposés sous les corniches des bas-côtés; les modillons à copeaux, inspirés du roman de l'église Saint-Pierre de Marnans (Isère), utilisés pour la première fois à la chapelle de La Salette en 1852 et à Champier en 1853, en plus sobres et faciles à mouler en ciment, placés sous les corniches de la nef centrale; les hautes bases en forme de piédestal biseautées au profil d'une doucine comme à La Salette et à Champier; un type de rosace moderne, dodécalobée, parfaitement adaptée à l'horloge.

\section{Le prix de l'achivement}

Le projet de Baudot avait été refusé pour Saint-Bruno car il était trop dispendieux. Baudot n'avait qu'une solution pour réduire les coûts " en remplaçant de la pierre de Sassenage par de la pierre de Beaucaire, et substituant le moellon enduit au mortier au moellon piqué de Sassenage pour les faces latérales " 8 . Pour lui comme pour Questel, en dehors d'un " dallage en carillottes de ciment moulé " ${ }^{89}$, point de salut dans le ciment à cette date : "Ce système de construction qui, au point de vue de l'économie peut présenter certains avantages nous parâ̂t infiniment regrettable pour un édifice de l'ordre de celui-ci, comme durée et comme effet. Ces pierres artificielles ne nous semblent pas donner des résultats satisfaisants ${ }^{90}$. "Tony Desjardins n'avait pas non plus d'autre avis sur la construction : "L'emploi du

84. 2 M 35, église Saint-Bruno de Grenoble, travaux exécutés par le sculpteur H. Ding, 1877.

85. 2 Fi 965, Alfred Berruyer, Plan modifie, eglise Saint-Bruno de Grenoble, plan au sol, $69,5 \times 48$, 25 septembre 1875. Le plan de Berruyer est refusé au profit de celui de Péronnet. Les piliers de Berruyer sont adoptés contre ceux de Durand ou de Péronnet.

86. Arch. dép. Isère, 2 O 185/27, Félix Breton, premier adjoint faisant fonction de maire, Extrait du registre des delibetrations du conseil municipal de Grenoble, 10 octobre 1875.

87. Arch. dép. Isère, 2 O 185/27, Gravier, du conseil des bâtiments civils; Pellat, du conseil de la préfecture; Dutey, voyer en chef du département; Lambert, voyer en chef honoraire; Alfred Berruyer, architecte diocésain, Avis sur la fleche de l'église Saint-Bruno de Grenoble, 5 avril 1878.

88. 2 M 29, Anatole de Baudot, architecte à Paris, rapport au maire de Grenoble, 6 février 1872.

89. 2 M 29, Anatole de Baudot, cahier des charges, église Saint-Bruno de Grenoble, 28 juin 1870.

90. 2 M 29, Charles Questel, conseil général des bâtiments civils, rapport sur la construction de l'église Saint-Bruno de Grenoble, 21 novembre 1873. 
ciment dans ces conditions est peu raisonné et [...] il n'y a pas à hésiter à employer de la pierre de taille lorsque le prix de revient n'est pas bien supérieur à celui du ciment $^{\text {'1." }}$

Depuis les années 1850, les architectes envoyés de Paris et notamment le premier diocésain de Grenoble Henri Janniard, faisaient des rapports positifs sur les ciments de l'Isère mais ces élèves des Beaux-Arts, classiques comme rationalistes, se préoccupaient de structure, de parement et non de matériaux moulés. Anatole de Baudot, proposait un coût de 342 francs le $\mathrm{m}^{2}$ pour une église en pierre de taille dure en 1870. Avec de la pierre de taille tendre, il comptait obtenir 315 francs le $\mathrm{m}^{2}$. Baudot avait calculé ses coûts en fonction des séries de prix hors propos. Il ne connaissait pas les carriers et n'avait pas compris que les entrepreneurs de Grenoble n'avaient pas l'habitude de gérer de tels chantiers. Péronnet lui-même dépassait les devis de son projet pourtant simple dès la mise en place des premières assises. Berruyer devait, lui, sommer Rayneri frères d'utiliser des pierres factices.

Les pierres factices de ciment moulé étaient utilisées de longue date en Isère, dans les angles des murs ou pour les encadrements mais rarement pour composer entièrement un mur, sauf ici. Rien de moderne dans la structure de l'édifice, rien de novateur, simplement les pièces moulées à bon creux sur le chantier et en petite série, en béton de ciment avec des galets roulés, ce qui permettait de réduire les coûts de construction du gros ouvre. L'entrepreneur Rayneri, dans le souci de réaliser quelques économies supplémentaires, utilisa la méthode industrielle des cimentiers Thorrand à Voreppe. Il se servait de ciment prompt naturel et de ciment lent artificiel mélangés pour confectionner des moulages en petit béton avec au centre un bloc de pierre non équarri servant de noyau. Le principe était d'ajouter des matériaux les moins onéreux en récupérant les déchets de pierres de carrière pour faire du remplissage dans le moule ${ }^{92}$. Cette méthode avait également modifié l'art du parement. D'ordinaire, les architectes dessinaient les moules que les entrepreneurs devaient confectionner eux-mêmes. Or Rayneri, avec ce même souci de rentabilité, acquit de la société Thorrand des moules en fonte crés pour la grande production $^{93}$. Il choisit deux modèles dont les parements étaient inconnus (ill. 6 et 7) :

1. Le moellon strié : des stries verticales d'un centimètre d'épaisseur espacées d'un centimètre, bordées d'un listel. 2. Le moellon quadrillé : une sorte d'opus reticulatum en miniature dont les carrés sur la pointe auraient deux centimètres de côté, bordé d'un listel ${ }^{94}$. Ces deux modèles étaient posés en alternance de telle sorte

91. 2 M 31, Tony Desjardins, architecte diocésain à Lyon; Louis Bresson, membre du conseil des bâtiments civils du Rhône, représentant l'organe des commissions réunies des travaux et du contenticux de Saint-Bruno de Grenoble, rapport au maire de Grenoble, 7 mai 1875.

92. Les carottages effectués par le L.R.M.H. montrent que ce procédé fut bien celui que la société Thorrand, Allard, Nicolet avaient propose dans son livret Ciments superieurs de Voreppe, Grenoble, Allier, 1878, fig. 22 et 23.

93. 2 M 31, Eugène Péronnet, lettre au maire de Grenoble, 2 février 1875.

94. Thorrand, Allard, Nicolet, Ciments supérieurs de Voreppe, op. cit., fig. 22 et 23. 


\section{Moëllons quadrilles}
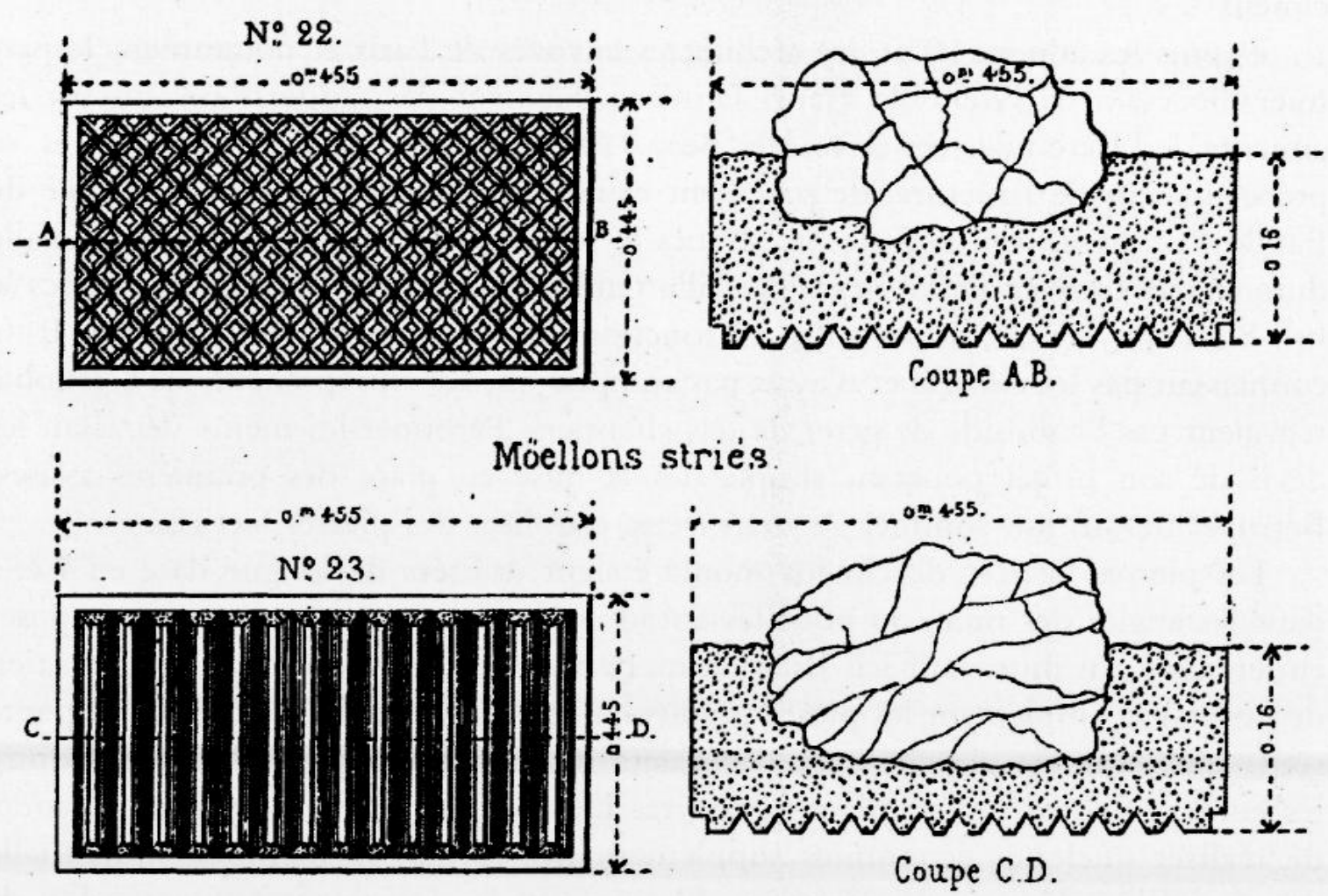

11l. 6 : Moellons quadrillés et stries, Thorrand, Allard, Nicolet, Ciments superieurs de Voreppe, Grenoble, Allier, 1878, fig. 22 et 23.

que les murs semblent, suivant la pénétration des rayons du soleil et la projection des ombres, alterner des assises claires et sombres rappelant non la trop byzantine cathédrale de Marseille de Léon Vaudoyer, mais la cathédrale de Gap par Charles Laisné.

L'eglise, travaux finis, coûta tout de même 552 francs le $\mathrm{m}^{2}$ : " Les travaux évalués a 300000 francs dans l'emprunt de $\mathbf{4 0 0 0 0 0 0}$ francs auront donc coûté 456777,21 francs. L'excédent aurait été bien plus considérable si les façades avaient été exécutées en pierre de taille, comme il était prévu dans les conditions imposées aux architectes lors du concours. Les dépenses auraient été au moins de 1000000 francs. C'est au moment de la mise à exécution de l'œuvre que l'on a reconnu la nécessité, par mesure d'économie, de construire cet édifice en pierres factices de béton et de ciment. De ce qui précède, il ressort un grand enseignement pour la période des grands travaux dans laquelle nous allons entrer $n^{95}$.

95. 2 M 32, Edouard Rey, maire de Grenoble, liquidation des dépenses relatives à l'église Saint-Bruno, 23 mai 1881. 


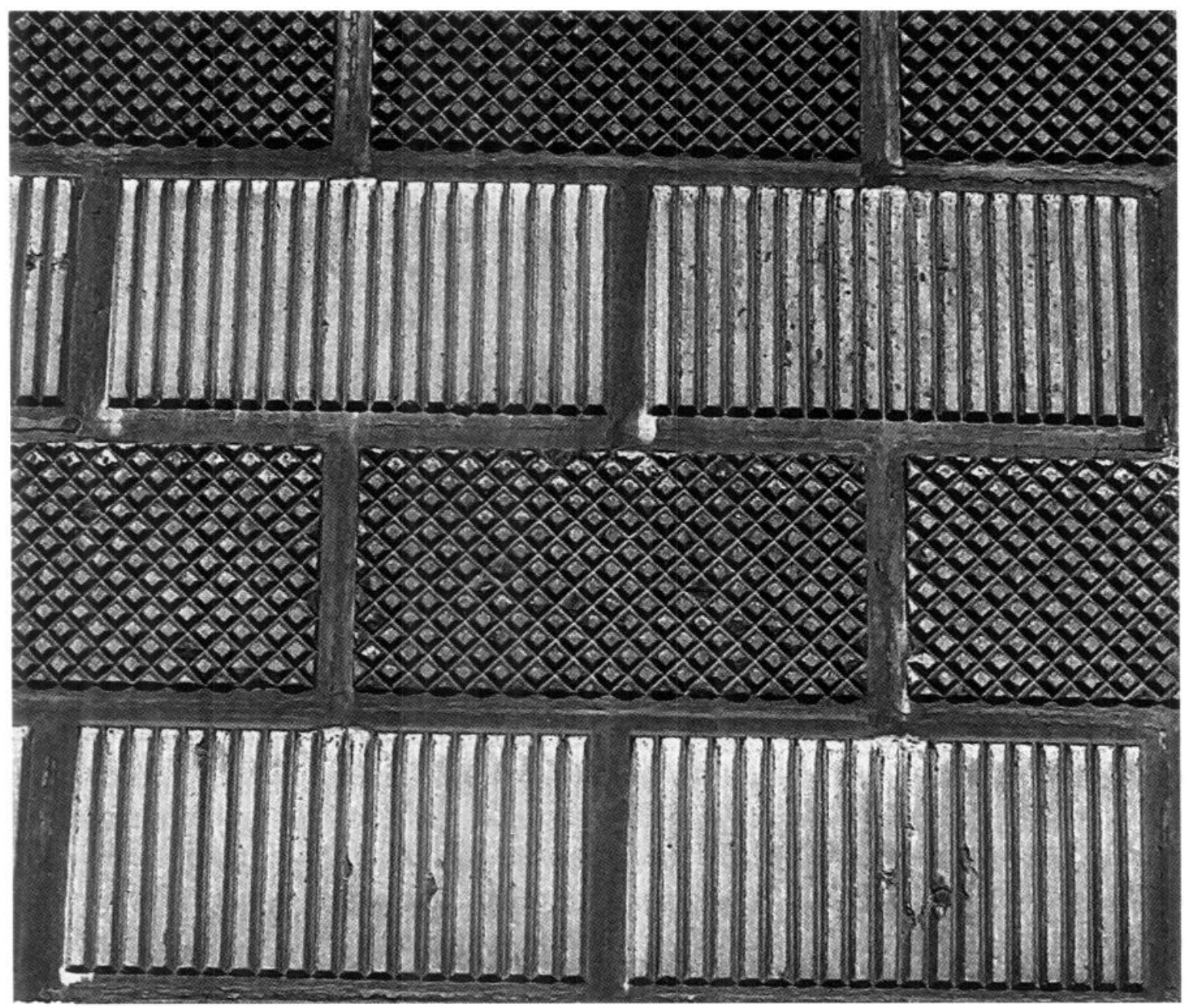

Ill. 7 : Parement de l'ćglise Saint-Bruno de Grenoble (1869-1881), détail. Cl. C. Avenier.

$\mathrm{Vu}$ les péripéties des chantiers et la qualité des acteurs de la construction, les églises de l'Isère avaient de quoi exciter les imaginations. Pourtant, malgré la simplicité de ces édifications en comparaison à la taille des grands chantiers du $\mathrm{XIX}^{\mathrm{e}}$ siècle, malgré l'organisation du réseau des constructeurs de l'Isère, retenons que ces édifices ne sont pas l'aboutissement de projets définis par leurs auteurs. L'imbroglio des desseins et de la gestion des chantiers s'oppose en tout aux grandes constructions publiques conçues et conduites depuis Paris à l'image des édifices de la place d'armes de Grenoble et, en particulier, la préfecture et le muséebibliothèque ${ }^{96}$. Les architectes ne pouvaient pas concevoir leurs projets sans prendre en compte la destination, l'emplacement, les programmes des maîtres d'ouvrages : les résolutions du maire de Voiron ou celui des avant-projets de Riondel dans le concours de Saint-Bruno de Grenoble sont symptomatiques. On constate aussi une part considérable d'imprévus : nouveaux plans de financements, repositionnement

96. Cyrille Simonnet, Le Musée-Bibliothèque de Grenoble. Histoire d'un projet, chroniques d'un chantier, Grenoble, P.U.G., 1987, 128 p. 
des conceptions de l'architecte, initiatives de l'entrepreneur, du maître d'ouvrage, aléas des chantiers, etc. Les projets d'architecture se sont construits en même temps qu'avançaient les chantiers. C'était de l'anti-architecture au sens académique et pourtant une réalité ordinaire.

Enfin, ces églises suivaient la voie de leurs contemporaines, inspirées par des œuvres maîtresses connues, et leurs systèmes d'architecture ne semblent pas à la hauteur de leur légende. Il ne s'agissait pas de copier servilement, non que cela manquât d'originalité mais parce que les œuvres procédant des théories rationalistes ou historicistes étaient trop complexes ou trop onéreuses. Les architectes bâtissaient dans le " style du XIII" siècle " ou du " roman-byzantin " mais la structure de leurs édifices restait banale et l'ornementation sobre, sans atteindre l'austérité de l' « Ecole diocésaine ". Saint-Bruno de Voiron comme de Saint-Bruno Grenoble ont bénéficié d'un système de construction fondé sur le souci d'économie lié à celui d'embellir. Leur muralité et la disposition des ouvertures créant une relation simple plan/ structure, structure/élévation offrent une lisibilité intérieure et extérieure directe. Les architectes, loin d'inventer un renouveau dans l'art, se concentraient sur une structure qu'on pourrait qualifier de rationaliste, si on interprète le rationalisme architectural non comme le système théorisé, déterminé, savant et reproduit en tant que gloire d'une époque ou d'une technique, mais comme celui de l'économie structurelle; un système ordinaire de maçonnerie, produit de l'agencement d'eléments récurrents, moulés, préfabriqués, un ensemble adapté aux besoins du culte, de l'urbanisme ainsi qu'aux ressources limitées et aux savoir-faire restreints : pas de commande prestigieuse, pas d'architecture audacieuse; pas d'édifices monumentaux, pas de technique monumentale; recours aux matériaux locaux, ciments compris. Le rationalisme, c'était l'adaptation des matériaux et des procédés mis à disposition par l'industrie : ici les ciments moulés furent les moteurs des variations d'ornementations, voire de structure. Ce rationalisme offre alors une autre valeur historique à ces deux églises Saint-Bruno, dépasse la question du style et complète la portée architecturale de l'œuvre pragmatique d'architectes comme Alfred Berruyer.

Cédric AVEnier

docteur en histoire de l'art chercheur-associé au C.R.H.I.P.A. (Université de Grenoble II Pierre-Mendès France) et au laboratoire Cultures constructives des Écoles d'architecture de Grenoble et Versailles 